University of Nebraska - Lincoln

DigitalCommons@University of Nebraska - Lincoln

2011

\title{
Aligned and exchange-coupled FePt-based films
}

Yi Liu

University of Nebraska-Lincoln, yliu@unl.edu

Thomas A. George

University of Nebraska-Lincoln, tgeorge1@unl.edu

Ralph Skomski

University of Nebraska-Lincoln, rskomski2@unl.edu

David J. Sellmyer

NCMN, dsellmyer@unl.edu

Follow this and additional works at: https://digitalcommons.unl.edu/physicssellmyer

Part of the Physics Commons

Liu, Yi; George, Thomas A.; Skomski, Ralph; and Sellmyer, David J., "Aligned and exchange-coupled FePtbased films" (2011). David Sellmyer Publications. 225.

https://digitalcommons.unl.edu/physicssellmyer/225

This Article is brought to you for free and open access by the Research Papers in Physics and Astronomy at DigitalCommons@University of Nebraska - Lincoln. It has been accepted for inclusion in David Sellmyer Publications by an authorized administrator of DigitalCommons@University of Nebraska - Lincoln. 


\title{
Aligned and exchange-coupled FePt-based films
}

\author{
Y. Liu, T. A. George, Ralph Skomski, and D. J. Sellmyer ${ }^{a)}$ \\ Department of Physics and Astronomy and Nebraska Center for Materials and Nanoscience, \\ University of Nebraska, Lincoln, Nebraska 68588, USA
}

(Received 16 June 2011; accepted 6 October 2011; published online 28 October 2011)

\begin{abstract}
Two-phase nanostructures of hard $L 1_{0}$-ordered FePt and soft iron-rich fcc Fe-Pt are investigated experimentally and by model calculations. The Fe-Pt thin films were produced by epitaxial co-sputtering onto $\mathrm{MgO}$ and have a thickness of about $10 \mathrm{~nm}$. They form two-phase dots that cover a large fraction of the surface but are separated from each other. X-ray diffraction and TEM show that the $c$-axis of the phase FePt is aligned in the direction normal to the film plane. The experimental and theoretical hysteresis loops indicate archetypical exchange coupling, and excellent magnetic properties are obtained. The largest values of coercivity, saturation magnetization, and nominal energy product obtained in the samples studied are $51 \mathrm{kOe}, 1287 \mathrm{emu} / \mathrm{cc}$, and 54 MGOe, respectively. (C) 2011 American Institute of Physics. [doi:10.1063/1.3656038]
\end{abstract}

The concept of achieving a useful high-energy-product magnet through appropriate nanostructuring of known hard and soft phases has been tantalizing researchers since the idea was first proposed. ${ }^{1-3}$ The field has received added impetus recently because of the potential scarcity of rare-earth metals used in today's best permanent magnets. ${ }^{4}$ The fabrication of exchange-coupled nanocomposite magnets faces several challenges. This includes (1) achieving a uniform mixture of hard and soft phases with a characteristic length scale of order $10 \mathrm{~nm}$, (2) arranging the nanostructure so that the coercivity remains high as the fraction of highmagnetization soft phase is increased, (3) aligning the easy anisotropy axes of the hard-phase grains, and (4) fabricating dense-packed bulk magnets for practical use. ${ }^{5-7}$ Similar problems have been tackled in magnetic recording. ${ }^{8-12}$

In permanent magnetism, randomly oriented anisotropy axes reduce the maximum energy product $(B H)_{\max }$ to somewhat more than one fourth of the "aligned" value. Thus, the $(B H)_{\max }$ values obtained for isotropic nanocomposite magnets thus far are limited to about 25 MGOe $\left[200 \mathrm{~kJ} / \mathrm{m}^{3}\right]$, which is about $40 \%$ of the best aligned $\mathrm{Nd}_{2} \mathrm{Fe}_{14} \mathrm{~B}$-type magnets. ${ }^{6,7}$ It has been possible to produce $L 1_{0}$-based single phase thin-film patches with excellent alignment and coercivity. ${ }^{13}$ But this has been difficult to achieve so far for $t w o-$ phase nanostructures.

Here, we investigate exchange-coupled $L 1_{0}$ FePt: A1 $\mathrm{Fe}-\mathrm{Pt}$ films grown on $\mathrm{MgO}$ (001) substrates. This enables the (001) growth of the $\mathrm{L} 1_{0} \mathrm{FePt}$, which has a reasonably high magnetization of $14.3 \mathrm{kG}$ [1.43 T] and a high anisotropy $K_{1}=66 \mathrm{Merg} / \mathrm{cm}^{3}\left[6.6 \mathrm{MJ} / \mathrm{m}^{3}\right]$. By increasing the Fe content beyond 50 atomic percent, the value corresponding to the ordered $L 1_{0}$ phase, we create an aligned coherent nanocomposite of ordered $L 1_{0}$ FePt and an Fe-rich fcc (A1) phase $[\mathrm{Fe}(\mathrm{Pt})]$. We analyze the magnetic properties of the nanocomposite as a function of the direction of the applied field.

The Fe-rich Fe-Pt films were deposited on (001) oriented single-crystal $\mathrm{MgO}$ substrates in an AJA International

\footnotetext{
${ }^{\text {a) }}$ Author to whom correspondence should be addressed. Electronic mail: dsellmyer@unl.edu.
}

sputtering system, with in-situ heating at $830^{\circ} \mathrm{C}$. The sputtering rate of $\mathrm{Fe}$ and $\mathrm{Pt}$ were adjusted for co-sputtering to realize various $\mathrm{Fe}$ concentrations. The compositions were calculated from the sputtering rates of $\mathrm{Fe}$ and $\mathrm{Pt}$, which were determined by $\mathrm{x}$-ray reflectivity measurements of the film thickness. Before each deposition, a vacuum of better than $4 \times 10^{-8}$ Torr was achieved and the Ar pressure during deposition was 5 mTorr. The films were characterized using a Rigaku x-ray diffractometer and a JEOL 2010 transmission electron microscope. The magnetic properties were measured using a quantum design SQUID magnetometer up to a maximum applied field of $70 \mathrm{kOe}$ at room temperature.

The nominal composition of the films is $\mathrm{Fe}_{\mathrm{x}} \mathrm{Pt}_{100-\mathrm{x}}$ with the following atomic percentages $x$ of Fe: (a) 59.4, (b) 60.6, (c) 62.8, and (d) 64.0. The samples exhibit similar x-ray diffraction patterns, with little or no change as a function of $\mathrm{Fe}$ concentration. Figure 1 shows the $\mathrm{x}$-ray diffraction peaks for the lowest $(x=59.4)$ and highest $(x=64.0)$ Fe contents. Only three strong peaks are observed: FePt (001), FePt (002), and $\mathrm{MgO}$ (002). The location of the FePt (111) peak is also indicated. This peak is the strongest one in FePt films without alignment, such as those deposited on a quartz

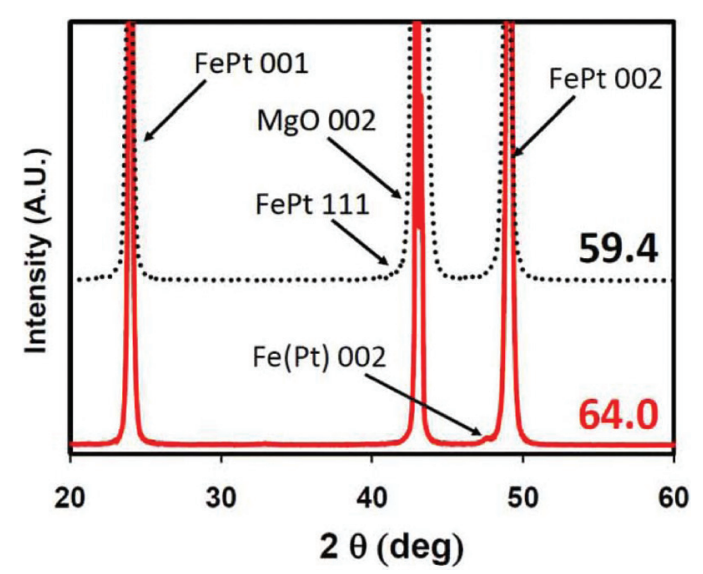

FIG. 1. (Color) X-ray diffraction patterns of $\mathrm{x}=59.4$ (dotted) and 64.0 (full) samples showing nearly perfect (001) FePt epitaxial growth on (001) $\mathrm{MgO}$. 
(a)

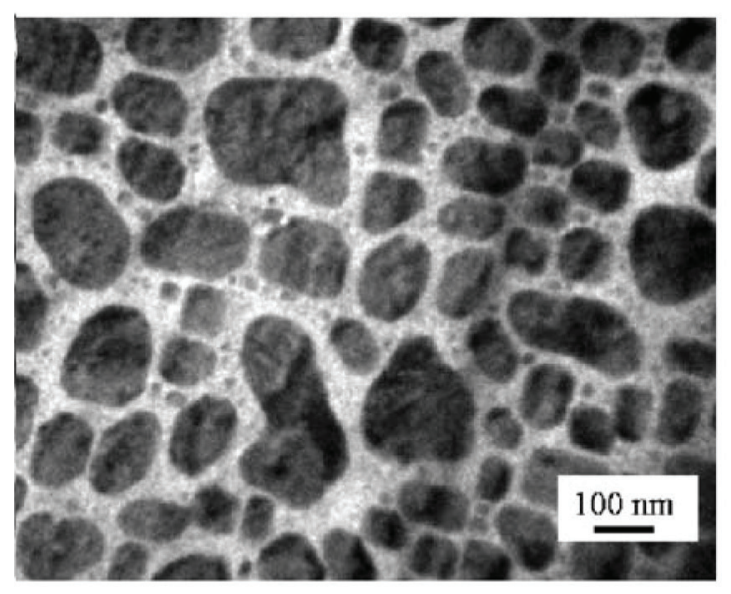

(b)

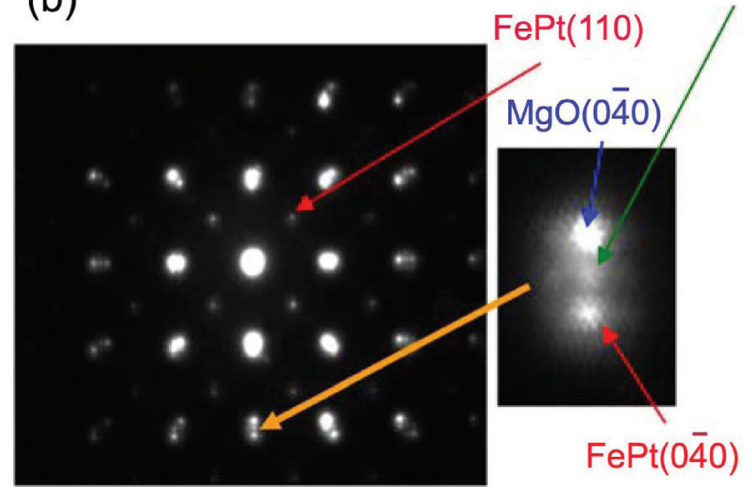

FIG. 2. (Color) Fe-rich Fe-Pt film on an MgO substrate: (a) TEM brightfield images and (b) [001] diffraction pattern.

substrate at room temperature, and its absence in our samples indicates a nearly perfect epitaxial growth of FePt (001) on $\mathrm{MgO}(001)$.

The morphology of the films is shown in Fig. 2. The volume of magnetic material in the films is estimated from the measured sputtering rates, and the nominal film thickness is $10 \mathrm{~nm}$ based on these rates. However, the high substrate temperature causes the Fe-Pt crystals to form islands, of average thickness $14 \mathrm{~nm}$ and packing density of about $70 \%$. Figure 2 shows the TEM image (a) and the diffraction pattern (b) of iron-rich Fe-Pt on $\mathrm{MgO}$. From the enlarged part of (b), we see that the main diffraction spots split into higher-order peaks and each of the three higher-order spots corresponds to one phase. The $L 1_{0}$ phase gives rise to the (011) super-lattice spots, and the spot between the substrate $\mathrm{MgO}$ and $\mathrm{FePt}$ spots is from a Fe-rich phase. The absence of (100)-type super-lattice diffraction spots indicates that the Fe-rich phase is an fcc random solid solution with $\mathrm{Pt}$. The location and shape of the soft fcc $\mathrm{Fe}(\mathrm{Pt})$ phase is not revealed due to the blurring effect of the supporting $\mathrm{MgO}$ substrate. The fcc $\mathrm{Fe}(\mathrm{Pt})$ has been imaged by HRTEM earlier in continuous FePt-based films. ${ }^{6}$ The fcc $\mathrm{Fe}(\mathrm{Pt})$ exists as a roughly circular grain of about $10 \mathrm{~nm}$ which is coherent with the FePt lattice.

Figure 3 shows the room-temperature hysteresis loops for the four samples, with fields applied in-plane $(\|)$ and perpendicular to the film plane $(\perp)$. The coercivity $H_{\mathrm{c}}$ decreases
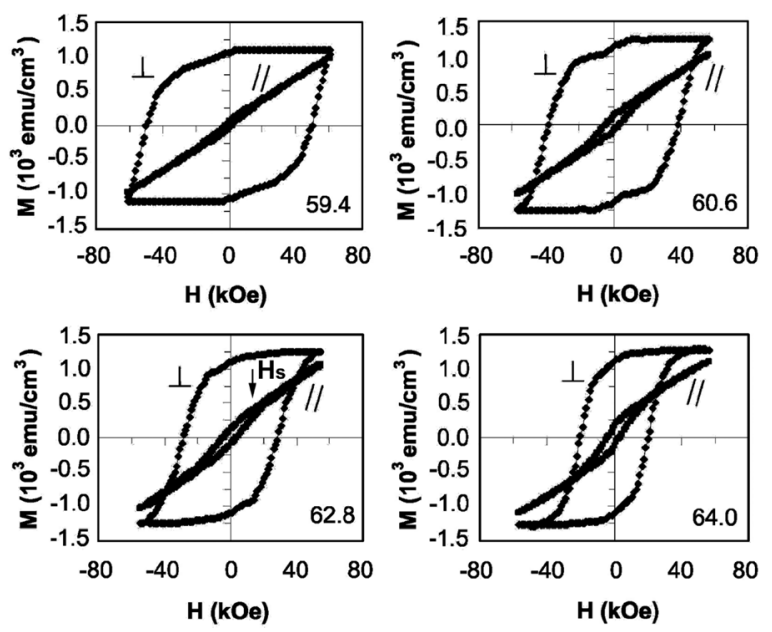

FIG. 3. Room temperature hysteresis loops of the Fe-Pt films with different Fe concentrations, as labelled. The in-plane and perpendicular loops are indicated by $(\|)$ and $(\perp)$, respectively. The field $H_{s}$ (see text) is indicated in $\mathrm{x}=62.8$ loop.

and the saturation magnetization $M_{\mathrm{s}}$ increases with increasing Fe concentration, as summarized in Table I. The in-plane and perpendicular loops of Fig. 3 are explained with the help of Fig. 4. In a macroscopic mixture of hard and soft magnetic phases, the zero-field slope (or susceptibility) $\chi_{\mathrm{s}}=\mathrm{d} M / \mathrm{d} H$ of the in-plane loop would be very large, ideally infinite. However, nanostructuring causes $\chi_{\mathrm{s}}$ to become finite and to depend on the dimensions of the soft regions, because the soft regions close to the hard-soft interface are effectively exchange-coupled to the hard phase. For perpendicular fields parallel to the $c$-axis, there is a clear magnetization drop at the soft-phase nucleation field $H_{n}$. While the $\mathrm{H}_{n}$ values in Fig. 3 appear to be nearly zero, when the loops are corrected approximately for demagnetization effects, $\mathrm{H}_{\mathrm{n}} \approx 9 \pm 1 \mathrm{kOe}$ for all samples.

Let us consider a field of the type $\mathbf{H}=H \cos \theta \mathbf{e}_{\mathrm{z}}+H$ $\sin \theta \mathbf{e}_{\mathrm{x}}$ and assume that the in-plane magnetization component $M_{\mathrm{x}}=M_{\mathrm{s}} \sin \phi$ of the soft phase is small, that is, $M_{\mathrm{z}}=M_{\mathrm{S}}$ $\phi(\mathbf{r})$. Neglecting magnetostatic selfinteractions, the micromagnetic energy functional is then

$$
E=\int\left(A(\nabla \phi)^{2}+\frac{\mu_{0} M_{s} H}{2} \cos \theta \phi^{2}(\mathbf{r})-\mu_{0} M_{s} H \sin \theta \phi(\mathbf{r})\right) \mathrm{d} V
$$

The eigenmodes of this functional are known for number of geometries, namely trigonometric, Bessel, and spherical Bessel functions for plate-like, cylindrical, and spherical inclusions. The initial susceptibility of the soft phase is given by

TABLE I. Room-temperature magnetic properties of $\mathrm{Pt}_{100-\mathrm{x}} \mathrm{Fe}_{\mathrm{x}}$ films. The uncertainties in the $M_{\mathrm{s}}$ and $(\mathrm{BH})$ measurements are estimated as $5 \%$ and $10 \%$, respectively.

\begin{tabular}{lccccc}
\hline \hline Sample & $\begin{array}{c}\mathrm{Fe} \\
(\text { at. \% })\end{array}$ & $\begin{array}{c}H_{\mathrm{c}} \\
(\mathrm{kOe})\end{array}$ & $\begin{array}{c}M_{\mathrm{s}} \\
(\mathrm{emu} / \mathrm{cc})\end{array}$ & $\begin{array}{c}(\mathrm{BH})_{\max }^{0} \\
(\mathrm{MGO})\end{array}$ & $\begin{array}{c}(\mathrm{BH})_{\max }^{\prime} \\
(\mathrm{MGOe})\end{array}$ \\
\hline (a) & 59.4 & 51.4 & 1216 & 40.0 & 52.9 \\
(b) & 60.6 & 38.5 & 1240 & 41.3 & 54.0 \\
(c) & 62.8 & 28.7 & 1267 & 39.5 & 52.1 \\
(d) & 64.0 & 20.1 & 1287 & 37.4 & 51.3 \\
\hline \hline
\end{tabular}




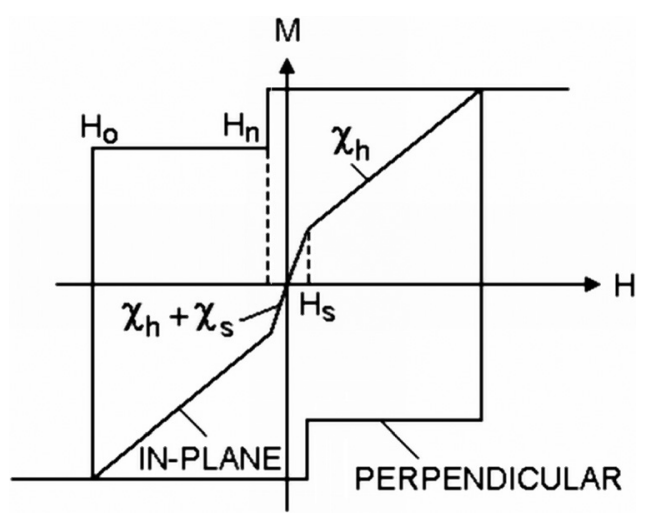

FIG. 4. Effect of exchange coupling on the hysteresis of hard-soft composites. Note that $H_{\mathrm{s}} / H_{\mathrm{n}} \approx 1.5$, depending on the geometry of the inclusion. $\chi_{\mathrm{h}}$ and $\chi_{\mathrm{s}}$ represent the slopes of the in-plane loop due to the hard and soft phase, respectively, and for macroscopic inclusions, $H_{\mathrm{s}}=H_{\mathrm{n}}=0$.

$$
\chi_{\mathrm{s}}=R^{2} / c_{o}^{2} l_{o}^{2}
$$

where $l_{\mathrm{o}}=\sqrt{A / 4 \pi M_{s}^{2}}$ is the proper exchange length and $c_{\mathrm{o}}=5.698$ for spherical inclusions, $c_{\mathrm{o}}=2.467$ for plate-like soft regions, and $c_{\mathrm{o}} \approx 4.1$ for cylindrical inclusions (columns normal to the film plane). $R$ is the radius of the spheres or cylinders, whereas embedded plates or films of thickness $t$ have $R=t / 2$ and soft patches on a hard surface are characterized by $R=t$. Determining $R$ from Eq. (2) and taking $H_{\mathrm{s}}=13 \mathrm{kOe}$ (from Fig. 3 ), $J_{\mathrm{s}}=18 \mathrm{kG}$, and $l_{\mathrm{o}}=2 \mathrm{~nm}$ yields $R=13.4 \mathrm{~nm}$ (spheres), $R=9.7 \mathrm{~nm}$ (cylinders), $t=5.8 \mathrm{~nm}$ (patches on hard surface), and $t=11.6 \mathrm{~nm}$ (embedded patches or layers).

The nucleation field of the soft phase can also be expressed in terms of $l_{\mathrm{o}}$

$$
H_{n}=2 M_{\mathrm{s}} x_{o}^{2} l_{o}^{2} / R^{2}
$$

Here, $x_{\mathrm{o}}$ is the first zero of the eigenmode, that is, $x_{\mathrm{o}}=\pi$ for the spherical Bessel function (spheres), $x_{\mathrm{o}}=\pi / 2$ for $\cos (x)$ (embedded plates), and $x_{\mathrm{o}}=2.4048$ for Bessel function (cylinders). Explicitly, the ratio $H_{\mathrm{n}} / M_{\mathrm{s}}$ is equal to $19.74 l_{\mathrm{o}}{ }^{2} / R^{2}$ (spheres), $11.57 l_{\mathrm{o}}^{2} / R^{2}$ (cylinders), $19.74 l_{\mathrm{o}}^{2} / t^{2}$ (embedded plates), and $4.94 l_{\mathrm{o}}{ }^{2} / t^{2}$ (flat patches on hard surface). Taking spherical inclusions, $J_{\mathrm{s}}=18 \mathrm{kG}$, and $H_{\mathrm{n}}=9 \mathrm{kOe}$ yields $R=12.5 \mathrm{~nm}$. This is reasonably close to the above value of $13.4 \mathrm{~nm}$ and means that the magnetization discontinuity in the perpendicular loop and the slope in the in-plane loop have the same origin, namely exchange coupling.

The fraction $f_{\mathrm{s}}$ of soft phase can be estimated by extrapolating the linearly increasing segment $M_{\|}\left(H>H_{\mathrm{s}}\right)$ to $H=0$. This yields $M_{\|}(0)=f_{\mathrm{s}} M_{\mathrm{ss}}$, where $M_{\mathrm{ss}}$ is the saturation magnetization of the soft phase. Assuming a value of $M_{\mathrm{ss}}$ appropriate to $\mathrm{Fe}_{3} \mathrm{Pt}$ of $1558 \mathrm{emu} / \mathrm{cm}^{3}$ gives $f_{\mathrm{s}}=4 \%$ for $x=59.4$ and $f_{\mathrm{s}}=16 \%$ for $x=64$. Thus, in these films, the soft phase is rather limited before the $\mathrm{H}_{\mathrm{c}}$ value drops significantly.

The energy product ${ }^{2}$ depends on the demagnetizing factor, which is an intricate matter for thin films, ${ }^{14}$ and on the packing fraction of the film. Here, we consider the nominal energy product, which ignores the voids between the Fe-Pt dots. However, Fig. 1 indicates that the dots can be quite densely packed, so that this is not a very serious limitation. Upper and lower bounds to the demagnetizing fields are obtained from uncorrected loops and from loops where the slope $\mathrm{d} M / \mathrm{d} H$ is constrained to diverge at $H_{\mathrm{c}}{ }^{13}$ Table I lists the respective uncorrected and corrected maximum energyproduct values $(B H)_{\max }^{0}$ and $(B H)_{\max }^{\prime}$. The maximum value of $(B H)_{\max }^{\prime}, 54$ MGOe for $x=60.6$, is as large as $89 \%$ of the theoretical maximum for this material $\left(4 \pi^{2} M_{\mathrm{s}}^{2}\right.$ or $\mu_{0} M_{\mathrm{s}}^{2} / 4$ in SI).

It is instructive to discuss the present films' excellent extrinsic properties (coercivity, magnetization, and energy product) in terms of grain isolation. The main problem with coercivity in two-phase nanostructures is that a single oversize soft inclusion may destroy the coercivity of a macroscopically large region. In other words, large soft regions create reverse domains that propagate through the magnet. Having relatively small magnetic two-phase particles, such as the big patches shown in Fig. 2(a), isolated by a nonmagnetic spacer, enhances the coercivity by suppressing the propagation of the domain wall into adjacent grains.

In conclusion, our results show that excellent extrinsic properties can be achieved in hard-soft two-phase nanostructures if the crystallite orientation and the feature size are controlled during processing. A factor contributing to the high coercivity and energy product of our Fe-Pt films is isolation between different two-phase dots, which suppresses the macroscopic expansion of magnetic domain walls. Such spatial hierarchy of small soft inclusions in larger and macroscopically arranged but isolated hard grains may also be a promising approach for other hard-soft nanostructures.

This work is supported by the U.S. Department of Energy, Office of Basic Energy Sciences, Division of Materials Sciences and Engineering under Award No. DE-FG0204ER46152 (film preparation, characterization, theory (YL, RS, and DJS), National Science Foundation Materials Science and Engineering Center under Award No. DMR0820521 (film preparation (TAG)), and the Nebraska Center for Materials and Nanoscience (Central Facility support). The authors are grateful to A. Kashyap, R. D. Kirby, S.-H. Liou, and P. K. Sahota for helpful discussions and to S. Michalski for experimental assistance.

${ }^{1}$ E. F. Kneller and R. Hawig, IEEE Trans. Mag. 27, 3588 (1991).

${ }^{2}$ R. Skomski and J. M. D. Coey, Phys. Rev. B 48, 15812 (1993).

${ }^{3}$ E. E. Fullerton, S. J. Jiang, and S. D. Bader, J. Magn. Magn. Mater. 200, 392 (1999).

${ }^{4}$ D. Kramer, Phys. Today 63(5), 22 (2010).

${ }^{5}$ D. J. Sellmyer, Nature 420, 374 (2002).

${ }^{6}$ W. Liu, Y. Liu, R. Skomski, D. J. Sellmyer in Handbook of Advanced Magnetic Materials, edited by Y. Liu, D. J. Sellmyer, and D. Shindo (Springer, Berlin 2006), Vol. 1, p. 182.

${ }^{7} \mathrm{~J}$. P. Liu in Nanoscale Magnetic Materials and Applications, edited by J. P. Liu, E. Fullerton, O. Gutfleisch, and D. J. Sellmyer (Springer, Berlin, 2009), p. 309.

${ }^{8}$ F. Casoli, F. Albertini, L. Nasi, S. Fabbrici, R. Cabassi, F. Bolzoni, and C. Bocchi, Appl. Phys. Lett. 92, 142506 (2008).

${ }^{9}$ T. A. George, Y. Yu, L. Yue, R. Skomski, and D. J. Sellmyer, IEEE Trans. Mag. 46, 2435 (2010).

${ }^{10}$ R. Skomski, T. A. George, and D. J. Sellmyer, J. Appl. Phys. 103, 07F531 (2008).

${ }^{11}$ V. Alexandris, Th. Speliotis, E. Manios, D. Niarchos, J. Fidler, J. Lee, and G. Varvaro, J. Appl. Phys. 109, $07 \mathrm{~B} 729$ (2011).

${ }^{12}$ B. Ma, H. Wang, H. Zhao, C. Sun, R. Acharya, and J. P. Wang, J. Appl. Phys. 109, 083967 (2011).

${ }^{13}$ T. Shima, K. Takanashi, Y. K. Takahashi, and K. Hono, Appl. Phys. Lett. 85, 2571 (2004).

${ }^{14}$ R. Skomski, Y. Liu, J. E. Shield, G. C. Hadjipanayis, and D. J. Sellmyer, J. Appl. Phys. 107, 09A739 (2010). 